\title{
Impairments in Episodic Future Thinking for Positive Events and Anticipatory Pleasure in Major Depression
}

\author{
Hallford, D. J. ${ }^{1,6}$, Barry, T. J. ${ }^{2}$, Austin, D. W. ${ }^{1}$, Raes, F. ${ }^{3}$, Takano, K. ${ }^{4}$, and Klein, B. ${ }^{5}$ \\ ${ }^{1}$ School of Psychology, Deakin University, 1 Gheringhap Street, Geelong, Victoria 3220, Melbourne, Australia \\ ${ }^{2}$ Department of Psychology, University of Hong Kong, Pok Fu Lam, Hong Kong \\ ${ }^{3}$ Faculty of Psychology and Educational Sciences, KU Leuven, Tiensestraat 102, Box 3712, 3000 Leuven, Belgium \\ ${ }^{4}$ Division of Clinical Psychology and Psychotherapy, Department of Psychology, Ludwig-Maximilians-University \\ Munich, Geschwister-Scholl-Platz 1, 80539 München, Germany \\ ${ }^{5}$ School of Health and Life Sciences, Federation University, University Dr, Mount Helen, VIC 3350, Australia \\ ${ }_{6}$ Corresponding Author, School of Psychology, Deakin University, 221 Burwood Hwy, Burwood, Victoria 3125, \\ Melbourne, Australia, Phone: + 6139251 7777, Email: david.hallford@deakin.edu.au
}

\section{Please cite as:}

Hallford, D. J., Barry, T. J., Austin, D. W., Raes, F., Takano, K., \& Klein, B. (2020). Impairments in episodic future thinking for positive events and anticipatory pleasure in major depression. Journal of Affective Disorders, 260, 536-543. https://doi.org/10.1016/j.jad.2019.09.039 


\begin{abstract}
Background: Characteristic of the cardinal symptom of anhedonia, people with clinical depression report lower levels of anticipatory pleasure. However, the psychological mechanisms underlying these deficits are poorly understood. This is the first study to assess whether, and to what extent, phenomenological characteristics of episodic future thinking for positive future events are associated with anticipatory pleasure among depressed individuals.
\end{abstract}

Methods: Individuals with a Major Depressive Episode (MDE; $N=117)$ and without $(N=47)$ completed ratings scales for depressive symptoms and trait anticipatory and consummatory pleasure. They then provided descriptions of personally-relevant positive future events and rated them for phenomenological characteristics and state anticipatory pleasure.

Results: Between-groups analysis showed that those with MDE reported lower trait anticipatory and consummatory pleasure. They also simulated future events with less specificity, less detail/vividness, less use of mental imagery, less use of first-person perspective, less plausibility/perceived likelihood of occurring, and reported less associated state anticipatory pleasure. In regression analyses in the depressed group, lower scores for detail/vividness, mental imagery, and personal significance all uniquely predicted lower state anticipatory pleasure.

Limitations: Cognitive functioning was not assessed, which may help clarify deficits that underpin these findings. History of previous depressive episodes in the comparison group were not assessed, which may underestimate between-group effects.

Conclusions: This study provides further evidence of deficits in anticipatory pleasure and episodic future thinking in depressed individuals. It also establishes links between state anticipatory pleasure and particular characteristics of episodic future thinking that may be amenable to intervention to reduce anhedonia.

Keywords: depression; episodic future thinking; anticipatory pleasure; anhedonia; episodic specificity

Word Count: 4983 
A hedonic response refers to the experience of pleasure arising from exposure to a rewarding stimulus or behaviour. As well experiencing pleasure from consuming reward, one can also experience anticipatory pleasure, that is, pleasure evoked from imagining rewarding experiences that might occur in one's future. For example, a person might experience pleasure in anticipation of spending time with friends, distinct from the experience later in the day when their friends are present. The pleasure that arises from anticipating an event is demonstrably distinct from consummatory pleasure that occurs during reward consumption (Berridge and Robinson, 2003; Kring and Caponigro, 2010), and is often stronger than the actual experience of emotion during the event that is being anticipated (Wilson and Gilbert, 2003). The adaptive importance of preexperiencing pleasure is apparent in its strong effect on decisions to approach or avoid a stimulus (Mellers et al., 1999; Treadway and Zald, 2011).

Anhedonia, or, difficulty in experiencing positive affect, is a cardinal feature of depression, and in particular is manifest in significantly lower levels of anticipatory pleasure (Hedge's $g=.87$ in comparisons of people with and without depression; Hallford and Sharma, 2019a). Low anticipatory pleasure is related to low engagement in rewarding behaviours (Engel et al., 2013; Sherdell et al., 2012), and contributes to overall psychosocial functioning (Foussias et al., 2011), however, the psychological mechanisms underlying the problems with anticipatory pleasure that people with depression experience are not well understood (Dunn, 2012). This is significant, as there is currently an unmet need in depression research in the study of pleasure and reward systems (Calabrese et al., 2014). More information is needed to further specify current treatments and develop new therapeutic options to target anhedonia in depression (Dunn, 2012).

Theories regarding the temporal experience of pleasure suggest that in order to preexperience pleasure a person has to be able to activate and maintain mental representations of the future experiences that one anticipates (Kring and Caponigro, 2010). In addition, simulations of future experiences that are more realistic and detailed are judged as being more likely to occur 
(Szpunar and Schacter, 2013), and are associated with stronger intentions to seek out and engage with these experiences (Tversky and Kahnemann, 1974). Compared to healthy people, people with depression show substantial difficulty with episodic future thinking, and in particular in generating specific and detailed representations of possible future events (Hallford et al., 2018). This difficulty in generating mental simulations of future events may be a factor in subsequent impairments in anticipatory pleasure. In particular, difficulty imagining positive future experiences may be associated with lower anticipatory pleasure and less motivation to seek out experiences that could lead to reward (Engel et al., 2013; Sherdell et al., 2012). Evidence indicates several characteristics of episodic future thinking for positive events that are associated with increased positive affect including the level of detail/vividness (Boland et al., 2018), use of imagery (Holmes et al., 2016), the use of first-person perspective (Holmes et al., 2008), the plausibility of the event (Szpunar and Schacter, 2013), and its personal significance (Barsics et al., 2016). Nevertheless, to the authors' knowledge, no study has explicitly assessed whether these characteristics of episodic future thinking are associated with anticipatory pleasure amongst people with depression. Knowing which characteristics of episodic future thinking contribute to deficits in anticipatory pleasure would be useful in specifying therapeutic techniques designed to treat anhedonia, and in particular to improve motivation and increase behavioural engagement in treatments such as behavioral activation.

Studies of anticipatory pleasure for personally-relevant events have high clinical relevance, as such events are proximal to the enactment of rewarding behaviours in the lives of depressed individuals (Engel et al., 2013; Sherdell et al., 2012). However, the majority of studies to date (see Hallford et al. 2018 for a systematic review and meta-analysis), have assessed trait tendencies to anticipate pleasure using the self-report Temporal Experience of Pleasure Scale (TEPS; Gard et al,. 2006; Li et al., 2015; Liu et al., 2014; Liu et al., 2017; Yang et al., 2014; Yang et al., 2017). Although the TEPS has utility in assessing group differences, its items involve abstract concepts that most likely also elicit an appraisal of one's current functioning rather than just the capacity to 
anticipate pleasure from future events (e.g., "I look forward to a lot of things in my life"). The TEPS also makes reference to concrete activities which may not be relevant for all respondents (e.g., "When I'm on my way to an amusement park, I can hardly wait to ride the roller coasters"). In contrast, state measures of anticipatory pleasure can assess responses to personally-relevant upcoming events, and examine specific characteristics of how the anticipated event is mentallysimulated that might predict the extent of a pleasure response. Only three studies have examined differences in state anticipatory pleasure in people with and without depression. MacLeod and Salaminiou (2001) found that people with depression, compared to those without, generated fewer positive events that they anticipated could happen to them, and reported less anticipated pleasure in relation to these events. Using the same paradigm, Bjärehed et al. (2010) found some support for an interaction effect whereby reduced anticipatory pleasure was observed in more immediate (i.e., next week), but not later (i.e., 5-10 years), timeframes, amongst people with depression. Lastly, Wu et al. (2017) asked participants over several days about upcoming events. They found that people with depression expressed significantly less anticipatory pleasure than healthy people for upcoming activities in daily life. The evidence to date therefore suggests that state anticipatory pleasure for specific upcoming events may be diminished in depression. However, further examination of episodic future thinking characteristics relating to these prospective events would provide more insight into which are most strongly associated with an anticipatory pleasure response.

The current study examined differences between people with and without depression regarding their self-reported trait and state anticipatory pleasure, as well as the phenomenological characteristics of episodic future thinking for personally-relevant, positive future events (i.e., detail/vividness, use of imagery, the use of first-person perspective, the plausibility of the event, and its personal significance). These phenomenological characteristics of episodic future thinking were then modeled as predictors in a regression equation to assess their contribution to reported anticipatory pleasure for these positive future events among those who were depressed. It was 
hypothesized that trait anticipatory and consummatory pleasure would be significantly lower in the group of individuals with depression relative to the control group. Further, that future thinking for personally-relevant positively-valenced events would be experienced with less detail/vividness, less use of mental imagery, less perceived plausibility, less use of first-person perspective, less personal significance, and less anticipatory pleasure in the group of individuals with depression relative to the control group. It was also hypothesized that in the depressed sample these characteristics would contribute towards lower levels of anticipatory pleasure for upcoming positive events.

\section{Methods}

\section{Participants}

Recent meta-analytic evidence shows large differences between people with and without depression in anticipatory pleasure (Hallford and Sharma, 2019). As such, we aimed to recruit at least 50 individuals to each group to be powered to detect at least medium sized between-groups effects, with $80 \%$ power and an alpha level of .05 . To detect a medium sized effect in anticipatory pleasure using the episodic future thinking predictors of interest in a multiple regression model, with $80 \%$ power and alpha level set at .05 , a sample size of at least 103 was required in the depressed group.

Participants were recruited online as part of a trial for a depression intervention through advertising on social media platforms (e.g. Facebook, Instagram), and various online groups and forums based in Australia. Participants were included in the depression group if they: had internet access in their home, were $\geq 18$ years of age, resided in Australia, were fluent in English, were experiencing a current Major Depressive Episode (MDE) as determined by the electronicPsychological Assessment System (e-PASS; see below; Nguyen et al., 2015) and if they had a score on the Patient Health Questionnaire (PHQ; Kroenke et al., 2010) of at least 10 indicating at least moderate depression. Participants were excluded if they indicated they were undergoing any current treatment for depression, with the exception of antidepressant medication that had been stable in 
type and dosage for at least four weeks. The first one hundred and seventeen individuals assessed for eligibility for the trial (Hallford et al. 2019) who met the depression criteria were included in this study. Forty-seven individuals who did not meet the criteria for depression on the e-PASS and had a score lower than five on the PHQ, indicating no depressive symptoms, were recruited for the control group using the same online methods. Therefore, these participants were a subsample of a group of participants who completed baseline measures as part of recruitment for a trial.

The age of the depressed group $(M=44.6, S D=13.4 ; N=117)$ was significantly higher than the non-depressed group $(M=39.4, S D=16.0 ; N=47), t(162)=2.0, p=.040, d=0.31$, and there were significantly more females in the depressed group (88\%) than the non-depressed group $(55.3 \%), \chi^{2}(1)=21.3, p<.001$. Regarding highest education level attained, the number of participants endorsing primary school (0.6\%), high school (20.7\%), and bachelor degrees (30.5\%) did not differ between the groups, but there were significant differences for diplomas/certificates (depression; 35.9\% versus non-depressed; $14.9 \%, p<.05$ ) and postgraduate degrees (depression; $14.5 \%$ versus non-depressed; $27.7 \%, p<.05)$.

\section{Materials}

Electronic Psychological Assessment System (e-PASS; Nguyen et al., 2015). The e-PASS is an online, self-report clinical assessment system, with items corresponding to a diagnosis of Major Depressive Episode from the Diagnostic and Statistical Manual of Mental Health Disorders IV-TR (DSM-IV-TR; American Psychological Association, 2000). The bereavement exclusion was removed to update the system to the DSM-5 criteria (American Psychological Association, 2013). Only the MDE module was used for this study, comprising 11 questions relating to the frequency of MDE symptoms over the last two weeks using a scale from 0 (not at all) to 4 (every day), and one item relating to severity using a 0 (no interference/distress) to 8 (extremely significant interference/distress). An algorithm is used to score the e-PASS in concordance with DSM criteria, whereby if participants endorse at least five symptoms as occurring either more days than not or 
every day, at least one of which must be disturbance in mood or anhedonia, and score three or more on the interference/distress question, they are categorised as experiencing an MDE. Diagnosis on the e-PASS for MDE has been shown to correspond well with diagnoses made using the structured, face-to-face Mini-International Neuropsychiatric Interview $(\kappa=.57$; Sheehan et al., 1998; Nguyen et al., 2015).

Patient Health Questionnaire-9 (PHQ-9; Kroenke et al., 2010). The PHQ-9 comprises nine self-report items referring to the Diagnostic and Statistical Manual for Mental Disorders $4^{\text {th }}$ edition text revision criteria for a Major Depressive Episode (American Psychiatric Association, 2000). Participants rate each item in reference to the frequency of the symptoms over the last two weeks on a scale of 0 (not at all) to 3 (nearly every day). The PHQ-9 has excellent psychometric properties for screening for probable depression, and in assessing the severity of depressive symptoms (Kroenke et al., 2010). The computerised version retains these excellent psychometric properties (Erbe et al., 2016). The internal reliability of the PHQ was good in the current study (Cronbach's $\alpha=.95)$.

Temporal Experience of Pleasure Scale (TEPS; Gard et al., 2006). The TEPS consists of 18 self-report questions that assess for trait anticipatory pleasure (10 items) and consummatory pleasure ( 8 items). Responses are given on a 6-point scale $(1=$ Very false for me to $6=$ Very true for $m e$ ), and items were averaged in each subscale so that higher scores indicated a stronger tendency to anticipate or experience pleasure. Internal reliability was found to be acceptable in the current sample for the anticipatory subscale (Cronbach's $\alpha$ across time-points $=.74$ ) and consummatory subscale (Cronbach's $\alpha$ across time-points $=.73$ ).

Episodic future thinking and state anticipatory pleasure. To assess the characteristics of episodic future thinking for positive events, participants were presented with five positive cue words (happy, strong, beautiful, easy, and hopeful) and instructions from the Episodic Future Thinking-Test (EFT-T; Hallford et al., inpress). No negatively-valenced cues were administered. 
Participants were asked to describe a different specific positive event that could happen in their future for each word. They were advised that the event did not have to be related to this word, but could merely be inspired by it. They were advised that it could happen at any time in their future, either soon or a very long time away. They were instructed that it must be something that was going to happen or could realistically happen, it must be something they would be personally involved in, and it must occur in a particular place and within the space of a day. They were asked to state as many details as they could about these possible future events, such as who was there, what would happen, how they would feel, and what they might think. They were provided with an example relating to the word pleasure. Each cue word was shown on a separate webpage. There was no time limit for the responses, but there was a minimum response of 25 characters needed for each cue so as to ensure responses were somewhat elaborated on. After describing the event, and before moving to the next word, they were asked a number of questions to assess the characteristics of the imagined experiences. Each of these questions were averaged across the cues to represent an overall score on these dimensions: detail/vividness (from 1; not at all to 5; very much so: internal reliability; $\alpha=.58$ ), the use of mental imagery (from 1; not at all to 5; very much so: internal reliability; $\alpha=.70$ ), the perspective it was imagined from (from -100; observer/third person perspective to +100 ; own eyes/first-person perspective: internal reliability; $\alpha=.76$ ), its significance/importance in relation to their personal goals (from 1; not at all to 5; very much so: internal reliability; $\alpha=.58$ ), and how plausible the experience seemed/how likely it would be to happen (from 1; not at all to 5; very much so: internal reliability; $\alpha=.55$ ). The temporal distance of the nominated future event was assessed for as well, as this is known to influence characteristics of the episodic future thinking relevant to anticipatory pleasure, such as detail/vividness and the sense of pre-experiencing a future event (D’Argembeau and Van der Linden, 2004). The scale used for this was, 1; in the next 24 hours, 2; in the next week, 3: in the next month, 4; in the next year, 5; in over a year, and 6 ; in over 10 years: (internal reliability; $\alpha=.61$ ). State anticipatory pleasure was 
assessed by asking participants to rate how pleasurable it was to think about the positive future event (from 1; not at all to 5; very much so: internal reliability; $\alpha=.69$ ). The written responses to the EFT-T items were also coded for specificity, as defined by the event being something that would happen within the space of a day $(1=$ specific, $0=$ non-specific $)$. The first author and another researcher (who was otherwise not affiliated with the study, and not explicitly told of the study design) coded approximately $20 \%$ of participants' responses on the EFT-T $(n=32)$ using a coding guide developed by Hallford et al. (inpress). Good inter-rater agreement was found $(\kappa=.87)$. The non-affiliated coder then coded the remaining responses. None of the written responses were nonsensical or obviously off-task, and only one response was missing from one cue word, which was coded as non-specific. These scores were then totaled for a score of episodic future thinking specificity. The internal reliability of the EFT-T items was acceptable $(\alpha=.64)$.

\section{Procedure}

The study received ethics approval from the Human Ethics Committee of the first author's University prior to commencing recruitment. Advertisements were posted online along with a link to the online survey that interested individuals could click on and be taken to a plain language statement. Participants with depression that endorsed items on the e-PASS indicating that they had a current MDE then went on to complete the measures as part of a larger battery of baseline assessments for the trial. For participants without depression, the same procedure was followed, but those who endorsed items on the e-PASS indicating they were not clinically depressed, and did not score over five on the PHQ, then continued to complete only the study measures and not the additional measures within the intervention trial battery. Informed consent was presumed by submission of the responses. No compensation was provided.

\section{Data Analysis}

SPSS 25.0 was used for all statistical analyses. To examine between-group differences on the variables of interest, while controlling for differences on the demographic variables of age, sex, 
and education level, ANCOVAs were used. Corresponding partial eta squared effect sizes of 0.01 were interpreted as small, 0.09 as medium, and 0.25 as large (Cohen, 1988). To control for type 1 errors, with $\alpha$ set at .05 , the results from the ANCOVA analyses were subject to the false discovery rate procedure (Benjamini \& Hochberg, 1995). Relative to multiple comparison corrections that control the familywise error rate to avoid making a single type I error, this procedure provides more statistical power to identify effects by aiming to control only the proportion of significant results that are type I errors. A corrected significance level is provided after the procedure $(q)$, with all $p$ values less than this level considered to be statistically significant.

A hierarchical multiple regression was conducted in the group of individuals with depression to assess how much unique variance the episodic future thinking characteristics predicted in state anticipatory pleasure for the imagined positive future event, after accounting for demographic variables, severity of depressive symptoms, and trait anticipatory and consummatory pleasure. Adjusted $R^{2}$ is reported as a measure of effect size, as this accounts for the number of predictors in the model by only increasing if a new predictor explains variance by more than would be expected by chance. The false discovery rate procedure was also applied to $p$-values for correlations and multiple regression weights.

\section{Results}

\section{Between-Groups Analyses}

Table 1 shows the descriptive statistics and the results of the ANCOVA analyses. As expected, the depressed group reported significantly higher depressive symptoms. The depressed group also reported significantly lower trait anticipatory and consummatory pleasure on the TEPS compared to the non-depressed group, to a large and moderate-to-large degree, respectively. The depressed group also reported significantly less state EFT-T anticipatory pleasure following episodic future thinking for positive events, with a moderate effect size. 
With respect to the characteristics of episodic future thinking for positive events assessed through the EFT-T, the depressed group described events that were significantly further in the future than the non-depressed group, with a moderate effect size. Compared to the non-depressed group, the depressed group reported fewer specific events on the EFT-T, to a small-to-moderate degree, less EFT-T detail/vividness, to a moderate-to-large degree, and reported less use of mental imagery on the EFT-T, to a small to moderate degree. Both groups reported a tendency to use first-person perspective on average for their episodic future thinking, but the depressed group endorsed this less strongly, to a moderate degree. No significant difference was found between the groups on how relevant the nominated positive future events on the EFT-T were in relation to their personal goals. The depressed group did, however, perceive the events reported on the EFT-T as being less plausible and less likely to happen than the non-depressed group, to a moderate-to-large degree. All analyses were repeated using ANOVAs without controlling for the demographic variables and the results did not differ.

\section{Correlational Analyses}

Turning to tests of linear associations in the depressed group, zero-order correlations indicated that all variables except for age, education, depressive symptom severity, temporal distance and perspective were associated with state anticipatory pleasure (see Table 2). It is noted that despite the lack of correlation with state anticipatory pleasure, higher depressive symptom severity was found to be related to lower trait anticipatory pleasure $(r=-.21, p=.022)$. At the first step of the hierarchical multiple regression, participants' age, sex, and education were entered as predictors. These variables predicted $4 \%$ of the adjusted variance in state anticipatory pleasure, and sex was the only variable which predicted significant unique variance $(\beta=.25)$. In the second step, symptom severity, and trait anticipatory and consummatory pleasure were entered. This resulted in an increase in predicted adjusted variance to $11 \%\left(\Delta R^{2}=7 \%\right)$, however, no variables were independent predictors following correction for multiple tests. In the last step, episodic future 
thinking characteristics were entered as predictors. The addition of these predictor variables within the model lead to a large increase in predicted variance in state anticipatory pleasure, totaling to $64 \%$ of the adjusted variance, which was significantly more variance than the second model $\left(\Delta R^{2}=\right.$ $53 \%)$. In this final model higher levels of detail/vividness $(\beta=.29)$, more use of mental imagery ( $\beta$ $=.33)$, and more significance of the event in relation to personal goals $(\beta=.24)$ predicted unique variance in state anticipatory pleasure. Other variables of age, education, depressive symptom severity, trait anticipatory and consummatory pleasure, episodic thinking specificity, temporal distance, perspective and plausibility of the future event did not contribute unique variance to state anticipatory pleasure.

\section{Discussion}

This study aimed to examine differences between depressed and non-depressed individuals on trait and state anticipatory pleasure, and the characteristics of episodic future thinking for positive future events. It is also the first study to assess whether and to what extent each of the characteristics of episodic future thinking for positive future events are associated with state anticipatory pleasure among depressed individuals.

The observations of lower trait anticipatory and consummatory pleasure in the depressed group replicated previous findings (Li et al., 2015; Liu et al., 2011; Liu et al., 2014; Yang et al., 2014; Yang et al., 2017), and further support the notion of overall reduced hedonic responses in depressed individuals. The group effect for trait anticipatory pleasure was substantially larger than for consummatory pleasure, indicating this group of people with depression perceived themselves as having a more pronounced deficit in expecting reward from future experiences relative to deriving pleasure from consuming rewards. Previous studies have reported mixed findings regarding differences in the relative magnitude of these deficits (e.g., Wu et al., 2017; Liu et al., 2011). Also consistent with our hypothesis, and other studies assessing state anticipatory pleasure (MacLeod and Salaminiou, 2001; Wu et al., 2017), participants with depression reported less 
anticipatory pleasure for upcoming positive events as part of the episodic future thinking task. This indicated that, in addition to a generalised deficit in anticipating pleasure, participants with depression experienced less state anticipatory pleasure when asked to describe specific, personallyrelevant events that could occur in their future.

Regarding the phenomenal characteristics of the future events that participants imagined, the group of depressed individuals reported positive future events as being less specific and less populated with details/less vivid, they involved less mental imagery, were less likely be in firstperson perspective, and were generally perceived as being less plausible/likely to occur. The group difference in episodic future thinking specificity is consistent with previous findings that depression is related to deficits in the ability to mentally-generate future events that are specific to a time and place (Hallford et al., 2018). The finding of reduced detail/vividness for positive events is also consistent with previous studies examining this characteristic in the context of depressive symptoms (e.g., Morina et al., 2011; Stöber, 2000). Although not all studies in this area have been consistent with this finding (e.g., Hach et al., 2014; Patron et al., 2015), the disparities between studies in this area may be due to some studies including positive, negative, and neutral future events (Hach et al., 2014; Patron et al., 2015), whereas the deficits in detail/vividness in depression appear to be specific to positive events (Holmes et al., 2016). The findings also support previous research that people with depression use less mental imagery (Holmes and Mathews, 2010), and more frequently use third-person perspective when mentally-representing experiences (Holmes et al., 2016; Kuyken and Howell, 2006; Kuyken and Moulds, 2009; Nelis et al., 2013). More use of mental imagery and first-person perspective are each associated with stronger emotional responses to mental representations of events (Holmes et al., 2016), and this is likely due to a stronger sense of preexperiencing. It has been suggested that depressed individuals tend to adopt a third-person, observer perspective in their episodic thinking to help down-regulate the distress that occurs from frequent, negatively-valenced thoughts (Williams and Moulds, 2007, 2008). This effect has also been found 
for positively-valenced episodic thinking (Nelis et al., 2013). This might also generalise to positive episodic future thinking, and lead to a blunted pleasure response when thinking of rewarding experiences. Finally, in the present study, participants with depression generated positive future events that were further away in time compared to the non-depressed group, consistent with some recent findings on future thinking in a dysphoric sample (Hallford, 2019) and with research indicating the retrieval of more remote positive autobiographical memories in clinical depression (Kim et al., 2018). Overall, depressed individuals, compared to non-depressed individuals, reported that positive events were represented in episodic future thinking in ways less likely to evoke positive affect or to be perceived as plausible.

In the group with depression, the characteristics of episodic future thinking were all correlated with state anticipatory pleasure, with the exception of specificity. When entered into the multiple linear regression, lower average scores for detail/vividness, mental imagery, and the significance of the event all uniquely predicted lower state anticipatory pleasure. Collectively, these variables predicted a substantial amount of variance in anticipatory pleasure. This supports the notion that those with depression have a paucity of rich mental representations of positive future events and that this may contribute to lower anticipatory pleasure. With respect to the personal significance of future thinking, the current study supports Barsics et al.'s (2016) findings in a nonclinical sample showing a positive correlation between the personal importance of future events and anticipatory pleasure, albeit they combined positive and negatively-valenced events in their analysis. It extends on these findings to show that this applies in people with depression, and that the personal significance of the future event predicts anticipatory pleasure independently of detail/vividness and the use of mental imagery. The temporal distance of the event was not found to be a significant predictor of anticipatory pleasure. The plausibility of the event was not a unique predictor in the model, indicating that specific ways in which the future event was represented in 
mind (with detail and use of imagery), and its significance, were more important in predicting reward-related response than how likely they were to happen.

Interestingly, trait anticipatory and consummatory pleasure only modestly correlated with state anticipatory pleasure, and neither were unique predictors of variance in the final model. This suggests that state anticipatory pleasure can fluctuate independently of the general disposition to experience hedonic responses, and that the expectation of reward from particular events is only partially predictable from generalised expectancies. In summary then, although people may possess an overall capacity for hedonic responses, there can also be variation in state anticipatory pleasure, and a large amount of variation in the latter can be predicted by how detailed/vivid their simulations of future events are, how much mental imagery is used, and how personally-significant the future experience seems.

Notably, state anticipatory pleasure was not correlated with depressive symptom severity in the depressed group, although trait anticipatory pleasure was. Given that the depressed group did report significantly lower state anticipatory than the non-depressed group, this suggests that the lower state anticipatory pleasure may be due to psychopathological processes of being depressed state rather than the particular severity of depression. Another explanation may be that variance within the depressed group on these variables was not sufficient to detect this association in state anticipatory pleasure.

This study provides the first evidence of a direct relationship between specific episodic future thinking characteristics and affective responses that arises from expectations of reward in those with depression. In addition to trait-level measures, future studies might focus on studying anticipatory pleasure and motivation in relation to specific upcoming events or behaviours, and whether they are subsequently engaged in. Episodic future thinking characteristics for these upcoming events can be examined as a causal factor if appropriate designs are used. Examining these variables for personally-relevant daily activities will provide a more fine-grained analysis of 
the interrelationship between the cognitive-affective mechanisms of approach-related, rewarding behaviour in depression. Including negative and neutral-valenced events may also be useful to contrast associations with positive-events. Another point to consider for future research is also examining anticipated pleasure, that is, the expectation that one will experience pleasure from an event when it occurs, in addition to the current experience of pleasure from anticipating the event. Although these concepts are theoretically intertwined, and empirically shown to be related (e.g., Barsics et al., 2016; Hallford et al., under review), a recent review provides some evidence that anticipated pleasure may reliably impact social behaviour and judgement (DeWall et al., 2016). Therefore, assessing how characteristics of episodic future thinking correlate with how depressed individuals perceive they will feel when experiencing something, in addition to how they currently feel when thinking about this experience, might provide further insight into mechanisms connecting cognition with behaviour.

Although those with depression appear to have difficulty accessing mental representations of positive future events in general (MacLeod and Salaminou, 2001), and in specific and detailed ways as demonstrated here, the contributing factors are not entirely clear, and may be many in number. Some possibilities may be a lack of motivation in generating events that stems from hopelessness, cognitive functioning difficulties (discussed below), or a higher frequency of ruminative thoughts that compete with cognitive resources for episodic thinking. Another possibility is that depressed individuals, given the propensity to think in a negatively-valenced way, may learn over time to avoid thinking about events in detail as a form of affect regulation. This form of functional avoidance, perhaps both in terms of specificity, detail and use of mental imagery, has been suggested to account somewhat for the development over overgeneral memory (Williams et al., 2007), and may be relevant in the context of future thinking. Moreover, impairments in memory may inhibit retrieval of content for the formation of rich mental representations of future events. 
With respect to clinical implications, the episodic future thinking characteristics that uniquely predict anticipatory pleasure are amenable to intervention. Indeed, non-dysphoric individuals and dysphoric individuals (Boland et al., 2018) can be guided to produce more detailed episodic future thinking for positive events. For individuals with depression, increases in episodic detail can be observed on problem-solving tasks following specificity inductions (MacFarland et al., 2017). Further, low-intensity activities can help to increase their use of detail and mental imagery for upcoming positive events (Hallford et al., under review), and for mental imagery in general (Blackwell et al., 2015). Notably, in this latter study those who reported more use of mental imagery also reported improved anhedonia, suggesting that experience of pleasure is impacted by increasing this particular dimension of episodic thinking (Blackwell et al., 2015). Although it is not known if such interventions have effects on anticipatory pleasure in particular, the current findings suggest that they would. Future studies should examine this possibility and in doing so they would also provide a test of the causal relations between the variables analyzed here. The findings also support the notion that episodic future thinking that is focused on events with greater relevance to personal goals will produce a stronger level of anticipatory pleasure, which may then generate a stronger motivation to engage in this rewarding behaviour (Sherdell et al., 2012). This is consistent with the notion of values-based techniques in therapies such as acceptance and commitment therapy (Hayes et al., 2011), and the behavioural activation components of Cognitive Behavioral Therapy (Hopko et al., 2003). Therefore, focusing on increasing detail/vividness and use of mental imagery, in the context of behaviours of high personal significance may produce the highest levels of anticipatory pleasure. Engaging in training to improve episodic future thinking and stimulate these characteristics, both in and out of session, may encourage a stronger reward response, increase motivation to engage in positive behaviours to experience consummatory pleasure, and encourage learning of these outcomes to improve psychosocial functioning. Indeed, evidence for using mental 
imagery as a 'motivation amplifier' to enact planned behaviours is already emerging (Renner et al., 2018), but requires examination in clinical populations.

There may also be a role for adjunct interventions to remediate deficits in episodic future thinking in depression. Adapting interventions such as memory specificity training (Raes, Williams, and Hermans, 2009), where depressed individuals effectively learn to mentally-generate specific, detailed past events, may be one method. Other studies in health behaviors provide evidence that incorporating such episodic future thinking activities with specific decision-making tasks would also improve depressed individuals' ability to choose larger, longer-term goals over smaller, immediate rewards (e.g., Snider et al., 2016; Sze et al., 2017). Indeed, depressed individuals have been shown to be more likely to discount delayed rewards at a higher rate than non-depressed (Pulcu et al., 2014), although the role of anticipatory pleasure in this difference has yet to be examined. Further, there may be possible transdiagnostic relevance for this approach to studying and improving aspects of episodic future thinking in other clinical groups that are known to have future thinking deficits, such as those with schizophrenia-spectrum, bipolar disorder (Hallford et al., 2018) and posttraumatic stress disorder (PTSD; Kleim et al., 2014). Indeed, similar characteristics of episodic future thinking examined in this study, or additional ones, might be linked with psychopathological processes that maintain illness in these disorders, such as reduced anticipatory pleasure in schizophrenia (Hallford and Sharma, 2019) and impaired problem-solving in PTSD (Zlomuzica et al., 2018).

The current study had some noteworthy limitations. The non-depressed group were not assessed for previous episodes of depression. This is significant, as differences between depressed and non-depressed individuals on trait anticipatory pleasure tend to be larger when ruling out a history of depression (Hallford and Sharma, 2019). Therefore, the group differences may be underestimated here. Executive functioning was not assessed, but has been found to predict some variance in future thinking specificity (Addis, Hach, and Tippett, 2016), and account for some 
differences between people with and without depression in memory specificity (e.g. Dalgleish et al., 2007). Notably though, deficits in executive functioning only appear to account partially for deficits in episodic thinking in depression (Addis et al, 2016; Dalgleish et al., 2007; Williams et al., 2007). Similarly, deficits in memory specificity that are found in depression (Williams et al., 2007) may be a factor in the impairments observed in episodic future thinking, but again only appear to account for a minority of this variance (Addis et al., 2016; Williams et al. 1996). Nonetheless, the assessment of these variables in future studies may help account for some observed findings .The prevalence of other mental health disorders, and those on psychotropic medications, were not assessed, but would have helped characterize the sample further, and may assist in delineating any effects of co-morbidities or medications in future studies. The internal reliability indices for the EFT-T items ranged from poor to acceptable, and therefore indicated some error in measurement which likely impacted on the findings. This may be due, in part, to the likely heterogeneity of the future events that were described on indices such as plausibility and personal significance, for which internal consistency was low. Examining whether the strength of associations between episodic future thinking characteristics and anticipatory pleasure differed between the depressed and non-depressed groups may also be of interest, and is a question for future studies with a large sample and adequate statistical power to conduct moderation analyses with this number of predictors. The time taken to describe events on the EFT-T was not assessed, and may have been a factor of interest in subsequent ratings on episodic future thinking characteristics and anticipatory pleasure. On a general note, these findings would benefit from replication in order to increase confidence in their robustness.

In conclusion, this study provides further evidence of deficits in anticipatory pleasure and episodic future thinking in depressed individuals. It establishes links between state anticipatory pleasure and particular characteristics of episodic future thinking. It also indicates particular aspects of episodic future thinking that may be amenable to intervention in order to treat anhedonia. 
Declarations of interest: None.

Funding: The authors received no funding from an external source. 


\section{References}

Addis, D. R., Hach, S., and Tippett, L. J. (2016). Do strategic processes contribute to the specificity of future simulation in depression?. British Journal of Clinical Psychology, 55(2), 167-186. doi:10.1111/bjc.12103

American Psychiatric Association. (2000). Diagnostic and statistical manual of mental disorders: $D S M-I V-T R$. Washington, DC: American Psychiatric Association

American Psychiatric Association. (2013). Diagnostic and statistical manual of mental disorders, 5th Edition (DSM-5). Washington, DC: American Psychiatric Association

Barsics, C., Van der Linden, M., and D'Argembeau, A. (2016). Frequency, characteristics, and perceived functions of emotional future thinking in daily life. The Quarterly Journal of Experimental Psychology, 69(2), 217-233. doi:10.1080/17470218.2015.1051560

Benjamini, Y., \& Hochberg, Y. (1995). Controlling the false discovery rate: a practical and powerful approach to multiple testing. Journal of the Royal Statistical Society: Series B (Methodological), 57, 289-300. doi:10.1111/j.2517-6161.1995.tb02031.x

Berridge, K. C., and Robinson, T. E. (2003). Parsing reward. Trends in Neurosciences, 26(9), 507-513. doi:10.1016/S0166-2236(03)00233-9

Bjärehed, J., Sarkohi, A., and Andersson, G. (2010). Less positive or more negative? Futuredirected thinking in mild to moderate depression. Cognitive Behaviour Therapy, 39(1), 3745. doi:10.1080/16506070902966926

Blackwell, S. E., Browning, M., Mathews, A., Pictet, A., Welch, J., Davies, J., ... and Holmes, E. A. (2015). Positive imagery-based cognitive bias modification as a web-based treatment tool for depressed adults: a randomized controlled trial. Clinical Psychological Science, 3(1), 91111. doi:10.1177/2167702614560746

Boland, J., Riggs, K. J., and Anderson, R. J. (2018). A brighter future: The effect of positive episodic simulation on future predictions in non-depressed, moderately dysphoric and highly 
dysphoric individuals. Behaviour Research and Therapy, 100, 7-16.

doi:10.1016/j.brat.2017.10.010

Calabrese, J. R., Fava, M., Garibaldi, G., Grunze, H., Krystal, A. D., Laughren, T., ... and Tohen, M. (2014). Methodological approaches and magnitude of the clinical unmet need associated with amotivation in mood disorders. Journal of Affective Disorders, 168, 439-451. doi: 10.1016/j.jad.2014.06.056

Cohen, J (1988) Statistical power analysis for the behavioral sciences (2nd ed.). Hillsdale, NJ: Erlbaum.

Dalgleish, T., Williams, J. M. G., Golden, A.-M. J., Perkins, N., Barrett, L. F., Barnard, P. J., . . . Watkins, E. (2007). Reduced specificity of autobiographical memory and depression: The role of executive control. Journal of Experimental Psychology: General, 136(1), 23-42. doi:10.1037/0096-3445.136.1.23

D’Argembeau, A., and Van der Linden, M. (2004). Phenomenal characteristics associated with projecting oneself back into the past and forward into the future: Influence of valence and temporal distance. Consciousness and Cognition, 13, 844-858. doi:10.1016/ j.concog.2004.07.007

DeWall, C. N., Baumeister, R. F., Chester, D. S., and Bushman, B. J. (2016). How often does currently felt emotion predict social behavior and judgment? A meta-analytic test of two theories. Emotion Review, 8(2), 136-143. doi:10.1177/1754073915572690

Dunn, B. D. (2012). Helping depressed clients reconnect to positive emotion experience: current insights and future directions. Clinical Psychology and Psychotherapy, 19(4), 326-340. doi: doi.org/10.1002/cpp.1799

Engel, M., Fritzsche, A., and Lincoln, T. M. (2013). Anticipatory pleasure and approach motivation in schizophrenia-like negative symptoms. Psychiatry Research, 210(2), 422-426. doi:10.1016/j.psychres.2013.07.025 
Erbe, D., Eichert, H. C., Rietz, C., and Ebert, D. (2016). Interformat reliability of the patient health questionnaire: Validation of the computerized version of the PHQ-9. Internet Interventions, 5, 1-4. doi:10.1016/j.invent.2016.06.006

Foussias, G., Mann, S., Zakzanis, K. K., van Reekum, R., Agid, O., and Remington, G. (2011). Prediction of longitudinal functional outcomes in schizophrenia: the impact of baseline motivational deficits. Schizophrenia Research, 132(1), 24-27. doi:10.1016/j.schres.2011. 06.026 .

Gard, D. E., Gard, M. G., Kring, A. M., and John, O. P. (2006). Anticipatory and consummatory components of the experience of pleasure: a scale development study. Journal of Research in Personality, 40(6), 1086-1102. doi:10.1016/j.jrp.2005.11.001

Gard, D. E., Kring, A. M., Gard, M. G., Horan, W. P., and Green, M. F. (2007). Anhedonia in schizophrenia: distinctions between anticipatory and consummatory pleasure. Schizophrenia Research, 93(1-3), 253-260. doi:10.1016/j.schres.2007.03.008

Gard, D. E., Gard, M. G., Kring, A. M., and John, O. P. (2006). Anticipatory and consummatory components of the experience of pleasure: a scale development study. Journal of Research in Personality, 40(6), 1086-1102. doi:10.1016/j.jrp.2005.11.001

Gard, D. E., Kring, A. M., Gard, M. G., Horan, W. P., and Green, M. F. (2007). Anhedonia in schizophrenia: distinctions between anticipatory and consummatory pleasure. Schizophrenia Research, 93(1-3), 253-260. doi:10.1016/j.schres.2007.03.008

Hach, S., Tippett, L. J., and Addis, D. R. (2014). Neural changes associated with the generation of specific past and future events in depression. Neuropsychologia, 65, 41-55. doi:10.1016/j.neuropsychologia.2014.10.003

Hallford, D. J., Austin, D. W., Takano, K., \& Raes, F. (2018). Psychopathology and episodic future thinking: A systematic review and meta-analysis of specificity and episodic detail. Behaviour Research and Therapy. 102, 42-51.doi:10.1016/j.brat. 2018.01.003 
Hallford, D. J. (2019). The Phenomenological Characteristics of Autobiographical Future Thinking in Dysphoric and Non-Dysphoric Individuals. Psychiatry Research. doi:10.1016/j.psychres.2018.12.100

Hallford, D. J., Austin, D. W., Takano, K., \& Raes, F. (inpress). Psychometric Evaluation of an Episodic Future Thinking Variant of the Autobiographical Memory Test (Episodic Future Thinking - Test; EFT-T). European Journal of Psychological Assessment. Inpress. Pre-print availabe at https://psyarxiv.com/5uyba/

Hallford, D. J., \& Sharma, M. K. (2019). Anticipatory Pleasure for Future Experiences in Schizophrenia-Spectrum Disorders and Major Depression: A Systematic Review and MetaAnalysis. British Journal of Clinical Psychology. https://doi.org/10.1111/bjc.12218

Hallford, D. J., Sharma, M. K., \& Austin, D. W. (Under Review). Increasing Anticipatory Pleasure in Major Depression through Enhancing Episodic Future Thinking: A Randomized SingleCase Series Trial. Under Review. Preprint available at https://psyarxiv.com/9uy42/

Hallford, D. J., Austin, D. W., Raes, F., \& Takano, K. (2019). Online Memory Specificity Training (c-MeST) for the Treatment of Major Depression: A Study Protocol for a Randomised Controlled Trial. BMJ Open. http://dx.doi.org/10.1136/bmjopen-2018-024508

Holmes, E. A., Blackwell, S. E., Heyes, S. B., Renner, F., and Raes, F. (2016). Mental imagery in depression: phenomenology, potential mechanisms, and treatment implications. Annual Review of Clinical Psychology, 12, 249-280. doi:10.1146/annurev-clinpsy-021815-092925

Holmes, E. A., and Mathews, A. (2010). Mental imagery in emotion and emotional disorders. Clinical Psychology Review, 30(3), 349-362. doi:10.1016/j.cpr.2010.01.001

Holmes, E. A., Coughtrey, A. E., and Connor, A. (2008). Looking through rose-tinted glasses? Imagery perspective and positive mood. Emotion, 8(6), 875-879. doi: 10.1037/a0013617. 
Hopko, D. R., Lejuez, C. W., Ruggiero, K. J., and Eifert, G. H. (2003). Contemporary behavioral activation treatments for depression: Procedures, principles, and progress. Clinical Psychology Review, 23(5), 699-717. doi:10.1016/S0272-7358(03)00070-9

Li, Y., Mou, X., Jiang, W., Yang, Z., Shen, X., Jin, Z., ... and Yuan, Y. (2015). A comparative study of anhedonia components between major depression and schizophrenia in Chinese populations. Annals of General Psychiatry, 14(1), 24. doi:10.1186/s12991-015-0061-3

Liu, W. H., Chan, R. C., Wang, L. Z., Huang, J., Cheung, E. F., Gong, Q. Y., and Gollan, J. K. (2011). Deficits in sustaining reward responses in subsyndromal and syndromal major depression. Progress in Neuro-Psychopharmacology and Biological Psychiatry, 35(4), 1045-1052. doi:10.1016/j.pnpbp.2011.02.018

Liu, W. H., Wang, L. Z., Shang, H. R., Shen, Y., Li, Z., Cheung, E. F., and Chan, R. C. (2014). The influence of anhedonia on feedback negativity in major depressive disorder. Neuropsychologia, 53, 213-220. doi:10.1016/j.neuropsychologia. 2013.11.023

Kim, D., Yoon, K. L., and Joormann, J. (2018). Remoteness and valence of autobiographical memory in depression. Cognitive Therapy and Research, 42(3), 230-235. doi:10.1007/s10608-017-9881-6

Kleim, B., Graham, B., Fihosy, S., Stott, R., and Ehlers, A. (2014). Reduced specificity in episodic future thinking in posttraumatic stress disorder. Clinical Psychological Science, 2(2), $165-$ 173. doi:10.1177/2167702613495199

Kring, A. M., and Caponigro, J. M. (2010). Emotion in schizophrenia: where feeling meets thinking. Current Directions in Psychological Science, 19(4), 255-259. doi:10.1177/0963721410377599 
Kroenke, K., Spitzer, R. L., Williams, J. B., and Löwe, B. (2010). The patient health questionnaire somatic, anxiety, and depressive symptom scales: a systematic review. General Hospital Psychiatry, 32(4), 345-359. doi: 10.1016/j.genhosppsych.2010.03.006

MacLeod, A. K., and Salaminiou, E. (2001). Reduced positive future-thinking in depression: Cognitive and affective factors. Cognition and Emotion, 15(1), 99-107. doi:10.1080/02699930125776

McFarland, C. P., Primosch, M., Maxson, C. M., and Stewart, B. T. (2017). Enhancing memory and imagination improves problem solving among individuals with depression. Memory \& Cognition, 45(6), 932-939. doi:10.3758/s13421-017-0706-3

Mellers, B., Schwartz, A., and Ritov, I. (1999). Emotion-based choice. Journal of Experimental Psychology: General, 128(3), 332-345. doi:10.1037/0096-3445.128.3.332

Morina, N., Deeprose, C., Pusowski, C., Schmid, M., and Holmes, E. A. (2011). Prospective mental imagery in patients with major depressive disorder or anxiety disorders. Journal of Anxiety Disorders, 25(8), 1032-1037. doi:10.1016/j.janxdis.2011.06.012

Nelis, S., Debeer, E., Holmes, E. A., and Raes, F. (2013). Dysphoric students show higher use of the observer perspective in their retrieval of positive versus negative autobiographical memories. Memory, 21(4), 423-430. doi:10.1080/09658211.2012.730530

Nguyen, D. P., Klein, B., Meyer, D., Austin, D. W., and Abbott, J. A. M. (2015). The diagnostic validity and reliability of an internet-based clinical assessment program for mental disorders. Journal of Medical Internet Research, 17(9). doi:10.2196/jmir.4195

Patron, E., Benvenuti, S. M., Favretto, G., Gasparotto, R., and Palomba, D. (2015). Depression is associated with increased vagal withdrawal during unpleasant emotional imagery after cardiac surgery. Autonomic Neuroscience, 189, 75-82. doi:10.1016/j.autneu.2015.02.002 
Pulcu, E., Trotter, P. D., Thomas, E. J., McFarquhar, M., Juhász, G., Sahakian, B. J., ... and Elliott, R. (2014). Temporal discounting in major depressive disorder. Psychological Medicine, 44(9), 1825-1834. doi:10.1017/S0033291713002584

Raes, F., Williams, J. M. G., and Hermans, D. (2009). Reducing cognitive vulnerability to depression: A preliminary investigation of MEmory Specificity Training (MEST) in inpatients with depressive symptomatology. Journal of Behavior Therapy and Experimental Psychiatry, 40(1), 24-38. doi:10.1016/j.jbtep.2008.03.001

Renner, F., Murphy, F. C., Ji, J. L., Manly, T., and Holmes, E. A. (2019). Mental imagery as a “motivational amplifier" to promote activities. Behaviour Research and Therapy, 114, 51 59. doi:10.1016/j.brat.2019.02.002

Sheehan, D. V., Lecrubier, Y., Sheehan, K. H., Amorim, P., Janavs, J., Weiller, E., ... and Dunbar, G. C. (1998). The Mini-International Neuropsychiatric Interview (MINI): the development and validation of a structured diagnostic psychiatric interview for DSM-IV and ICD-10. The Journal of Clinical Psychiatry, 59(Suppl 20), 22-33.

Sherdell, L., Waugh, C. E., and Gotlib, I. H. (2012). Anticipatory pleasure predicts motivation for reward in major depression. Journal of Abnormal Psychology, 121(1), 51-60. doi:10.1037/a0024945

Snider, S. E., LaConte, S. M., and Bickel, W. K. (2016). Episodic future thinking: Expansion of the temporal window in individuals with alcohol dependence. Alcoholism: Clinical and Experimental Research, 40(7), 1558-1566. doi:10.1111/acer.13112

Stöber, J. (2000). Prospective cognitions in anxiety and depression: Replication and methodological extension. Cognition and Emotion, 14(5), 725-729. doi:10.1080/02699930050117693

Sze, Y. Y., Stein, J. S., Bickel, W. K., Paluch, R. A., and Epstein, L. H. (2017). Bleak present, bright future: online episodic future thinking, scarcity, delay discounting, and food demand. Clinical Psychological Science, 5(4), 683-697. doi:10.1177/2167702617696511 
Hayes, S. C., Strosahl, K. D., and Wilson, K. G. (2011). Acceptance and commitment therapy: The process and practice of mindful change. Guilford Press.

Szpunar, K. K., and Schacter, D. L. (2013). Get real: Effects of repeated simulation and emotion on the perceived plausibility of future experiences. Journal of Experimental Psychology: General, 142(2), 323-327. doi:10.1037/a0028877

Treadway, M. T., and Zald, D. H. (2011). Reconsidering anhedonia in depression: lessons from translational neuroscience. Neuroscience and Biobehavioral Reviews, 35(3), 537-555. doi:10.1016/j.neubiorev.2010.06.006

Tversky, A., and Kahneman, D. (1974). Judgment under uncertainty: Heuristics and biases. Science, 185(4157), 1124-1131. doi:10.1126/science.185.4157.1124

Williams, J. M. G., Barnhofer, T., Crane, C., Herman, D., Raes, F., Watkins, E., and Dalgleish, T. (2007). Autobiographical memory specificity and emotional disorder. Psychological Bulletin, 133(1), 122-148. doi:10.1037/0033-2909.133.1.122

Williams, A. D., and Moulds, M. L. (2007). Cognitive avoidance of intrusive memories: recall vantage perspectives associations with depression. Behaviour Research and Therapy, 45, 145-53. doi:10.1016/j.brat.2006.09.005

Williams, A. D., and Moulds, M. L. (2008). Manipulating recall vantage perspective of intrusive memories in dysphoria. Memory, 16, 742-750. 10.1080/09658210802290453

Wilson, T. D., and Gilbert, D. T. (2003). Affective forecasting. Advances in Experimental Social Psychology, 35(35), 345-411. doi:10.1016/s0065-2601(03)01006-2

Wu, H., Mata, J., Furman, D. J., Whitmer, A. J., Gotlib, I. H., and Thompson, R. J. (2017). Anticipatory and consummatory pleasure and displeasure in major depressive disorder: An experience sampling study. Journal of Abnormal Psychology, 126(2), 149-159. doi:10.1037/abn0000244 
Yang, X. H., Huang, J., Zhu, C. Y., Wang, Y. F., Cheung, E. F., Chan, R. C., and Xie, G. R. (2014). Motivational deficits in effort-based decision making in individuals with subsyndromal depression, first-episode and remitted depression patients. Psychiatry Research, 220(3), 874-882. doi:10.1016/j.psychres.2014.08.056

Yang, X. H., Tian, K., Wang, D. F., Wang, Y., Cheung, E. F., Xie, G. R., and Chan, R. C. (2017). Anhedonia correlates with abnormal functional connectivity of the superior temporal gyrus and the caudate nucleus in patients with first-episode drug-naive major depressive disorder. Journal of Affective Disorders, 218, 284-290. doi:10.1016/j.jad.2017.04.053

Zlomuzica, A., Woud, M. L., Machulska, A., Kleimt, K., Dietrich, L., Wolf, O. T., ... \& Margraf, J. (2018). Deficits in episodic memory and mental time travel in patients with post-traumatic stress disorder. Progress in Neuro-Psychopharmacology and Biological Psychiatry, 83, 4254. doi:10.1016/j.pnpbp.2017.12.014 
Table 1.

Descriptive Statistics and Results of Between-Groups ANCOVAs Controlling for Demographic Variables

\begin{tabular}{|c|c|c|c|c|c|}
\hline & Depressed $(n=117)$ & Non-Depressed $(n=47)$ & $F$ & $p$ & $\eta_{p}^{2}$ \\
\hline PHQ & $17.45(3.0)$ & $1.85(1.4)$ & 898.9 & $<.001$ & 0.85 \\
\hline TEPS-A & $2.94(0.80)$ & $4.14(0.98)$ & 60.5 & $<.001$ & 0.27 \\
\hline TEPS-C & $3.77(0.99)$ & $4.58(1.1)$ & 21.7 & $<.001$ & 0.12 \\
\hline EFT-T specificity & $2.00(1.53)$ & $2.70(1.51)$ & 4.2 & .038 & 0.02 \\
\hline EFT-T temporal distance & $3.96(0.86)$ & $3.35(0.98)$ & 17.6 & $<.001$ & 0.10 \\
\hline EFT-T detail/vividness & $3.23(0.68)$ & $3.70(0.79)$ & 19.6 & $<.001$ & 0.11 \\
\hline EFT-T imagery & $3.21(0.81)$ & $3.63(0.91)$ & 9.0 & .003 & 0.05 \\
\hline EFT-T perspective & $19.5(46.3)$ & $50.1(40.6)$ & 15.1 & $<.001$ & 0.08 \\
\hline EFT-T significance & $3.68(0.75)$ & $3.52(0.79)$ & 0.2 & .592 & 0.00 \\
\hline EFT-T plausibility & $3.23(0.71)$ & $4.08(0.70)$ & 47.1 & $<.001$ & 0.22 \\
\hline EFT-T anticipatory pleasure & $3.36(.79)$ & $3.75(0.78)$ & 14.2 & $<.001$ & 0.08 \\
\hline \multicolumn{6}{|c|}{$\begin{array}{l}\text { Note: the corrected significance level following the false discovery rate procedure was } q=.045 \text {, } \\
\text { with all } p \text {-values under this level considered to be statistically significant. PHQ = Patient Health }\end{array}$} \\
\hline \multicolumn{6}{|c|}{ Questionnaire, TEPS-A = Temporal Experience of Pleasure Anticipatory Pleasure Subscale, TEPS-c } \\
\hline
\end{tabular}


Running head: DEPRESSION FUTURE THINKING

Table 2.

Results of a Three-Step Hierarchical Multiple Regression Predicting State Anticipatory Pleasure in the Depressed Group ( $N=117)$

\begin{tabular}{|c|c|c|c|c|c|c|c|c|c|c|c|c|c|c|}
\hline $\begin{array}{l}\text { Zero-order } \\
r \text { with state } \\
\text { anticipatory } \\
\text { pleasure }\end{array}$ & Predictor & $r$ & $B$ & $S E B$ & $\beta$ & $s r^{2}$ & $B$ & $S E B$ & $\beta$ & $s r^{2}$ & $B$ & $S E B$ & $\beta$ & $s r^{2}$ \\
\hline & $\underline{\text { Step } 1}$ & & & & & & & & & & & & & \\
\hline .02 & Age & .02 & .00 & .00 & .03 & .00 & .00 & .00 & .03 & .00 & -.00 & .00 & -.03 & .00 \\
\hline $.25^{* *}$ & Sex & $.25^{* *}$ & .61 & .22 & $.25^{*}$ & .06 & .52 & .21 & .21 & .04 & .31 & .15 & .12 & .01 \\
\hline \multirow[t]{2}{*}{.07} & Education & .07 & .03 & .07 & .04 & .00 & .00 & .07 & .01 & .00 & -.05 & .04 & -.06 & .00 \\
\hline & $\underline{\text { Step } 2}$ & & & & & & & & & & & & & \\
\hline .04 & PHQ & .04 & & & & & .02 & .02 & .08 & .00 & .00 & .01 & .03 & .00 \\
\hline $.24 * *$ & TEPS-A & $.24 * *$ & & & & & .13 & .10 & .14 & .02 & -.01 & .06 & -.01 & .00 \\
\hline \multirow[t]{2}{*}{$.29 * *$} & TEPS-C & $.29 * *$ & & & & & .16 & .08 & .20 & .04 & .05 & .05 & .07 & .00 \\
\hline & $\underline{\text { Step } 3}$ & & & & & & & & & & & & & \\
\hline .16 & $\begin{array}{l}\text { EFT-T temporal } \\
\text { distance }\end{array}$ & .16 & & & & & & & & & .15 & .06 & .16 & .01 \\
\hline .03 & EFT-T specificity & .03 & & & & & & & & & -.02 & .03 & -.05 & .00 \\
\hline $.71 * * *$ & $\begin{array}{l}\text { EFT-T } \\
\text { detail/vividness }\end{array}$ & $\begin{array}{l}.71 * * \\
*\end{array}$ & & & & & & & & & .34 & .11 & $.29 * *$ & .02 \\
\hline $.69 * * *$ & EFT-T imagery & $.69 * *$ & & & & & & & & & .32 & .09 & $.33 * * *$ & .04 \\
\hline .15 & EFT-T perspective & .15 & & & & & & & & & .00 & .00 & -.02 & .00 \\
\hline $.59 * * *$ & EFT-T significance & $\begin{array}{l}.59^{* *} \\
*\end{array}$ & & & & & & & & & .25 & .07 & $.24 * *$ & .04 \\
\hline \multirow[t]{2}{*}{$.38^{* * *}$} & EFT-T plausibility & $\begin{array}{l}.38^{* *} \\
*\end{array}$ & & & & & & & & & .06 & .08 & .05 & .00 \\
\hline & $F$ for change in $R^{2}$ & & & & & $2.7 *$ & & & & $3.7 *$ & & & & $24.2 * * *$ \\
\hline
\end{tabular}




\section{DEPRESSION FUTURE THINKING}

Adjusted $R^{2}$

Note: ${ }^{*} p<.05,{ }^{* *} p<.01,{ }^{* *} p<.001 . s r^{2}=$ semi-partial correlation squared, indicating amount of unique variance between a predictor and anticipatory pleasure, not including variance

accounted for by other predictors. PHQ $=$ Patient Health Questionnaire, TEPS-A $=$ Temporal Experience of Pleasure Anticipatory Pleasure Subscale, TEPS-c $=$ Temporal Experience of Pleasure Consummatory Pleasure Subscale, EFT-T $=$ Episodic Future Thinking-Test The corrected significance level following the false discovery rate procedure for zero-order correlations was $q=.026$, with all $p$-values under this level considered to be statistically significant. The corrected significance rates for 